\title{
Wertvoll, aber aufwendig
}

Bruno Kesseli (Text und Bilder)

Dr. med. et lic. phil., Chefredaktor

Guidelines und Choosing-wisely-Listen sind sinnvoller Bestandteil einer guten Medizin: Darin waren sich die Teilnehmerinnen und Teilnehmer eines Workshops, der am 28. April in Bern stattfand, überwiegend einig. An Diskussionsstoff mangelte es trotzdem nicht, werfen doch Ausarbeitung und Anwendung dieser Instrumente eine Reihe von Fragen auf. Organisiert hatten den Anlass die Schweizerische Akademie der Medizinischen Wissenschaften SAMW und die Schweizerische Akademie für Qualität in der Medizin SAQM.

In letzter Zeit ist es etwas einfacher geworden, in der hausärztlichen Sprechstunde einem Patienten oder einer Patientin vorzuschlagen, angesichts der geschilderten Symptomatik zunächst einmal den Spontanverlauf zu beobachten, also eigentlich «nichts» zu tun. Denn Kampagnen, wie sie im vergangenen Jahr in der Schweiz von der Schweizerischen Gesellschaft für Allgemeine Innere Medizin SGIM unter dem Schlagwort «Smarter Medicine» lanciert worden sind, haben mittlerweile dank beachtlicher Resonanz in grossen Publikumsmedien weite Teile der Bevölkerung erreicht. Mit einem Klick auf die «Top-5-Liste» unter www.smarter medicine.ch kann man Skeptikern gleich im Untersuchungszimmer zeigen, dass SGIM, SAMW und der Dachverband Schweizerischer Patientenstellen davon abraten, schon nach drei Tagen leichter Kreuzschmerzen ein Röntgenbild, geschweige denn das gewünschte MRI zu machen. Dies erleichtert die Unterbindung des nach wie vor verbreiteten Reflexes «Ich erhalte keine
Intervention, also werde ich nicht ernst genommen» nicht unwesentlich.

\section{Qualität als integraler Bestandteil medizinischen Handelns}

Der Workshop von SAMW und SAMQ - der eigentlich eher ein klassisches Symposium mit Vorträgen und Diskussionsrunden war als eine Arbeitstagung - fokussierte neben Choosing-wisely-Listen (CWL) mit überflüssigen oder schädlichen medizinischen Massnahmen auch auf medizinische Guidelines. Christoph Bosshard, für die SAQM zuständiges Mitglied des Zentralvorstands der FMH, unterstrich in seiner Begrüssungsansprache, dass Qualität integraler Bestandteil des medizinischen Handelns sei. Mehr Transparenz sei in diesem Bereich indes wünschenswert, und gerade in Bezug auf die Implementierung und Anwendung von Guidelines bestünden viele Unsicherheiten. Er rief die

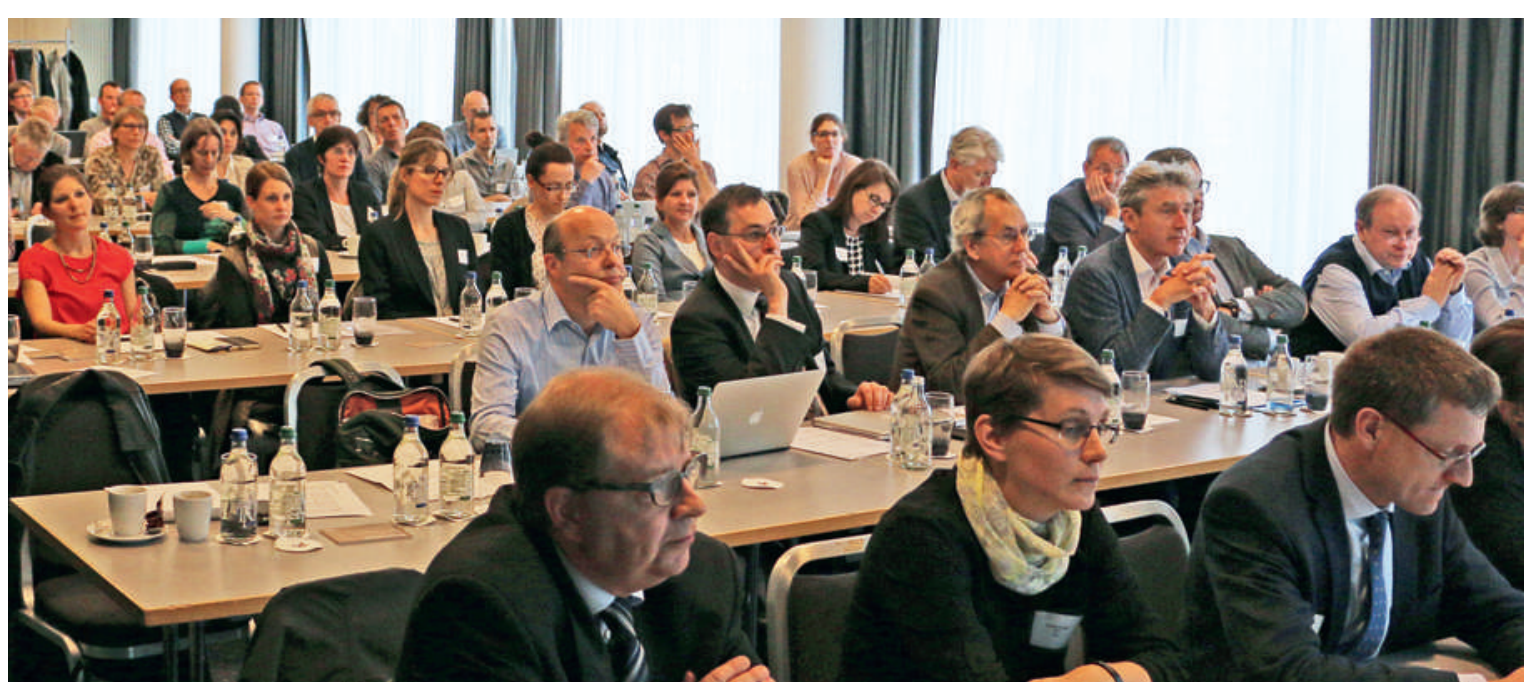

Das fachkundige Publikum folgte den Ausführungen der Referenten interessiert und diskutierte engagiert mit. 


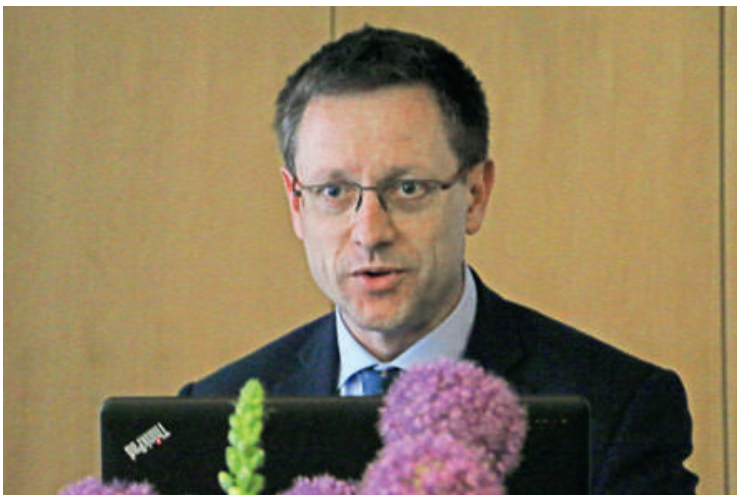

Christoph Bosshard

Position der FMH in Erinnerung, die qualitativ hochstehenden medizinischen Guidelines eine zentrale Bedeutung für die Sicherstellung der Behandlungsqualität beimisst, sie aber als «typisierende Empfehlungen» einstuft, die nicht jedem Einzelfall gerecht werden können. Auch die SAMW hat sich die Förderung von Guidelines und Choosing-wisely-Listen auf die Fahnen geschrieben und diese Zielsetzung bereits 2012 in ihrem Positionspapier Nachhaltige Medizin festgehalten [1]. SAMW-Generalsekretär Hermann Amstad präsentierte zum Auftakt der Tagung die Ergebnisse einer Umfrage, die die Akademie im November 2014 bei 44 Fachgesellschaften, 14 Ärztenetzwerken, 17 grossen Spitälern und 5 nichtärztlichen Berufsverbänden gemacht hatte. «Welches sind aus Ihrer Sicht die grössten Probleme bei der Ausarbeitung von Guidelines und Choosingwisely-Listen?», lautete die übergeordnete Frage, auf die mit einem Raster von 20 Unterfragen Antworten gesucht wurden. Bei einer Rücklaufquote von $54 \%$ darf den Ergebnissen durchaus eine gewisse Aussagekraft zugebilligt werden. Es zeigte sich, dass der Nutzen von Guidelines weitgehend unbestritten ist, während die Choosing-wisely-Listen nicht ganz so überzeugend abschnitten. Allerdings gaben nur drei der 24 befragten Fachgesellschaften und eines von sechs Netzwerken an, solche Listen seien unnötig oder irrelevant. Wenn

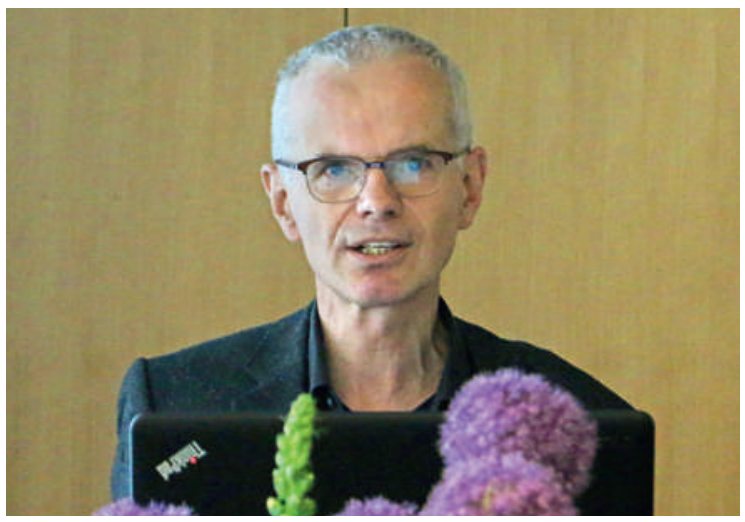

Hermann Amstad die befragten Organisationen mehrheitlich auf die Ausarbeitung eigener Listen verzichten, liegt dies gemäss den Umfrageergebnissen überwiegend an fehlenden personellen, fachlichen und finanziellen Ressourcen. Auch bei den Guidelines wird der entsprechende Aufwand als sehr hoch eingestuft.

\section{Der lange Weg zur Top-5-Liste}

Dass Choosing-wisely-Listen in der jüngeren Vergangenheit ein beachtetes und diskutiertes Thema sind, hängt zweifellos mit der angesprochenen Kampagne der SGIM zusammen. Nicolas Rodondi, Chefarzt und Leiter der Poliklinik am Inselspital Bern, zeichnete in seinem Referat den langen Weg zur Top-5-Liste nach, einer Zusammenstellung von fünf diagnostischen oder therapeutischen Interventionen, auf die in einem näher bezeichneten Kontext verzichtet werden sollte. Rodondi wies darauf hin, dass gemäss seriösen Schätzungen in

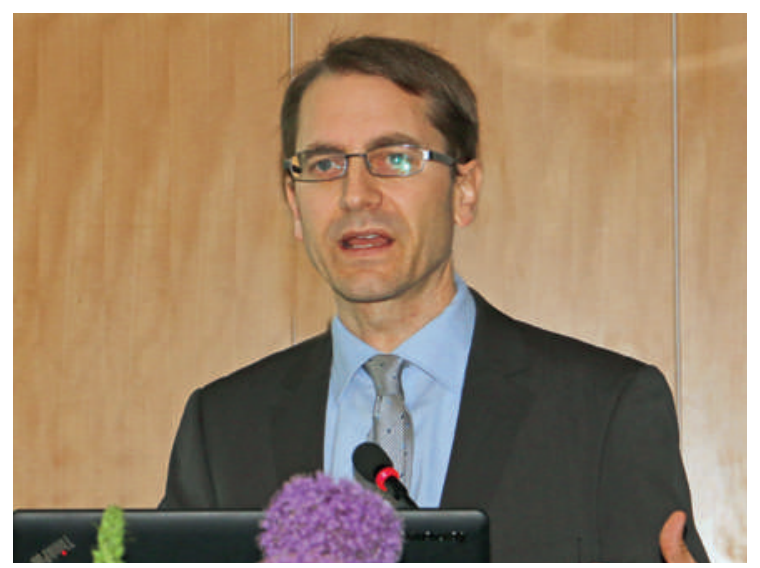

Nicolas Rodondi

den USA 30 Prozent der Gesundheitsausgaben durch unnötige Leistungen verursacht werden, und vermutete für die Schweiz ähnliche Zahlen. Seine Ausführungen verdeutlichten aber auch, dass der Weg zu fundierten CWL in der Tat sehr aufwendig ist, ein Punkt, der auch für Guidelines gilt und im Verlauf der Tagung in den Diskussionen immer wieder zur Sprache kam.

In die Ausarbeitung der Top-5-Liste waren eine Gruppe von 35 Experten und ein siebenköpfigen Steuerungskomitee involviert. Gemeinsam erarbeiteten sie in einem dreistufigen Delphi-Prozess die Empfehlungen und publizierten die Methodik auch in einem Journal mit Peer Review [2]. Für Rodondi ist klar, dass Top-5-Listen ein wichtiges Instrument der Kommunikation mit den Patienten sind und auch von weiteren Fachgesellschaften erarbeitet werden sollten. In den USA hätten dies bereits mehr als 60 Fachgesellschaften getan. Es sei essentiell, dass der Lead in Bezug auf die Wahl diagnostischer und therapeutischer Interventionen bei der 


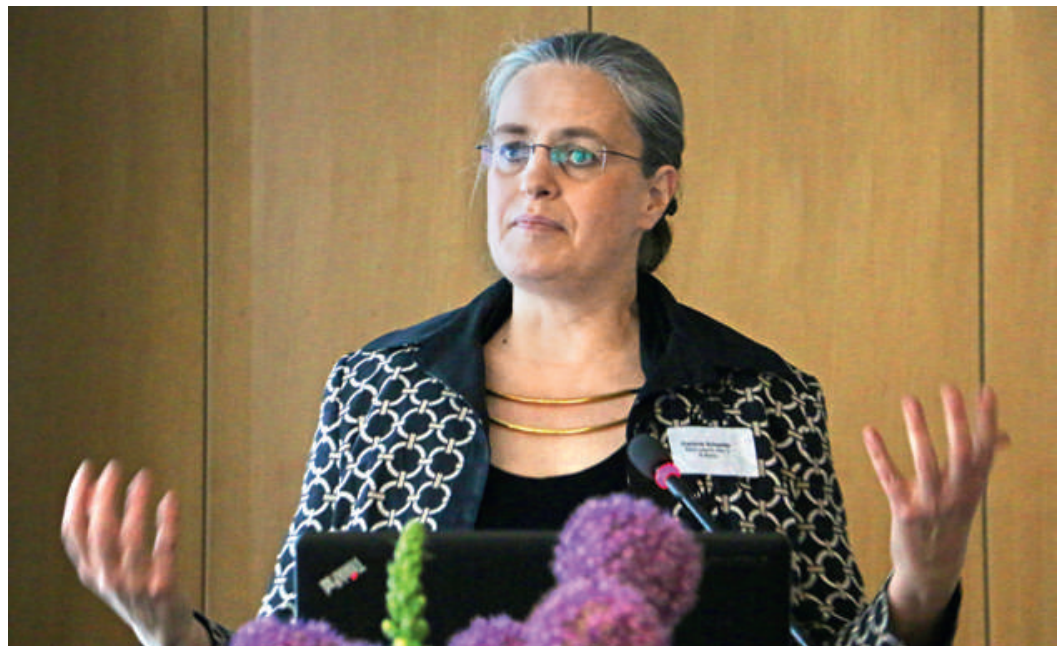

Corinna Schaefer stellte das «deutsche Modell» vor.

Ärzteschaft bleibe, und ein Engagement in diesem Bereich könne dazu beitragen.

\section{Lernen vom deutschen Modell}

Das «deutsche Modell» für medizinische Leitlinien stellte Corinna Schaefer vom Ärztlichen Zentrum für Qualität in der Medizin (ÄZQ) in Berlin vor. Man darf grundsätzlich feststellen, dass die Bemühungen um eine gewisse Ordnung im Guideline-Dschungel nördlich des Rheins weiter gediehen sind als hierzulande. Das ÄZQ ist das gemeinsame Kompetenzzentrum von Bundesärztekammer und Kassenärztlicher Bundesvereinigung für Qualität und Wissenstransfer im Gesundheitswesen. Einer seiner Aufgabenschwerpunkte ist die Entwicklung, Bewertung, Verbreitung und Methodik medizinischer Leitlinien.

Schaefer zeigte in ihrem Referat eindrücklich, mit welchen Herausforderungen der Transfer von medizinischem Wissen verbunden ist. Die Halbwertszeit des Wissens wird immer kürzer - bereits nach fünf Jahren ist die Hälfte dessen, was Medizinstudierende heute lernen, entweder überholt oder falsch. Belief sich die Zahl der medizinischen Neupublikationen Mitte des 20. Jahrhunderts noch auf einige Tausend pro Jahr, so werden heute täglich rund 75 neue klinische Studien und 11 systematische Reviews publiziert. Gemäss einer Schätzung des Deutschen Ärzteblatts haben durchschnittliche Mediziner aber lediglich 20 Minuten pro Woche für das Studium von Fachliteratur zur Verfügung. Das ist $(\mathrm{zu})$ wenig, aber mit Lesen allein wäre es ohnehin nicht getan. Um wissenschaftliche Artikel oder den Wert von Studien beurteilen zu können, ist Methodenkompetenz erforderlich, die in sehr vielen Fällen nicht gegeben ist. Und selbst wenn man die hätte, bleibt immer noch das Problem, dass rund die
Hälfte aller Studien wegen unliebsamer Ergebnisse gar nicht veröffentlicht wird und dass positive Ergebnisse erheblich früher publiziert werden als negative. Unter solchen Voraussetzungen macht es nachvollziehbarerweise Sinn, dass ein nationales Kompetenzzentrum mit entsprechenden Kapazitäten die Aufgabe übernimmt, die Spreu vom Weizen zu trennen.

Auch in Deutschland obliegt die Leitlinienentwicklung den einzelnen Fachgesellschaften, was eine «Krautund-Rüben-Situation» begünstigt. Dieser Tendenz wirkt indessen die Arbeitsgemeinschaft der Wissenschaftlichen Medizinischen Fachgesellschaften (AWMF) entgegen, die Unterstützung bietet bei Fragen der Bereitstellung, Methodik, Koordination und Klassifikation von Leitlinien. Und es gibt mittlerweile mit dem «Programm für Nationale VersorgungsLeitlinien (NVL)» und dem «Leitlinienprogramm Onkologie» zwei nationale Leitlinienprogramme mit formalen und methodischen Vorgaben, deren Koordination, Moderation und Durchführung durch das ÄZQ erfolgt.

Die Stärken der aktuell zwölf nationalen Leitlinien, deren Entwicklungszeit im Durchschnitt 3,5 Jahre betrug, liegen gemäss Schaefer in der methodischen Stringenz, der Evidenzbasierung und im unmittelbaren «Impact» auf die Versorgung. Weitere Vorzüge seien die Transparenz, der Einbezug aller relevanten Stakeholder sowie die Darstellung von Dissens und die Möglichkeit zu «Sondervoten». Als Herausforderungen nannte sie die hohen Kosten der Entwicklungsprozesse, die Komplexität der Materie, die Implementierung und Vertrauensfragen aufgrund möglicher Interessenskonflikte der involvierten Experten.

\section{Würdigung bisheriger Anstrengungen}

Einige dieser "Herausforderungen» waren bereits im vorangehenden Referat von Markus Trutmann angesprochen worden, der in seiner Funktion als Generalsekretär die Position der fmCh erläuterte, des Dachverbands von 16 chirurgisch und invasiv tätigen Fachgesellschaften. Gemäss Trutmann anerkennt die $\mathrm{fmCh}$, die über ihr Engagement in Sachen Guidelines noch nicht abschliessend entschieden hat, den Nutzen dieses Instruments durchaus, sieht aber verschiedene Probleme. Die Erarbeitung internationaler Guidelines wird als sehr aufwendig beurteilt, während bei nationalen Leitlinien die Gefahr der Beeinflussung durch Partikularinteressen gross sei. Als schwierig wird von der fmCh auch die Frage taxiert, wer über die Gültigkeit von Guidelines entscheidet und in welcher Weise diese aktualisiert oder allenfalls widerrufen werden. Zudem wies Trutmann darauf hin, dass in der Chirurgie nur 6-8 Prozent der Leistungen evidenzbasiert 


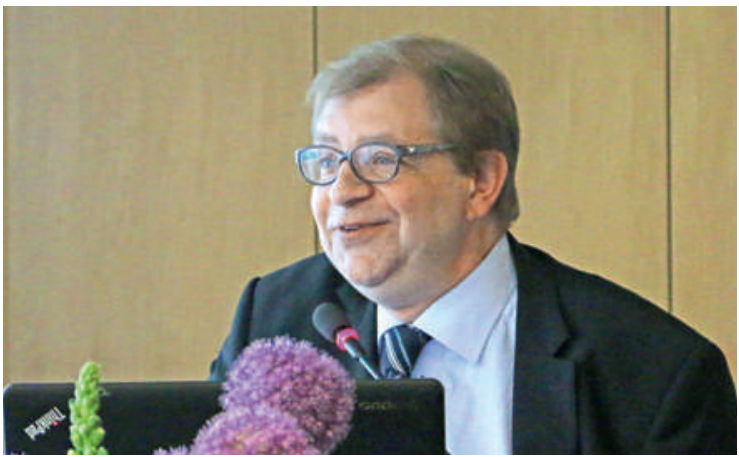

Markus Trutmann

seien. Die fmCh sieht «multidisziplinäre Boards», beispielsweise präoperative Tumorboards und inter-institutionelle Indikationsboards, als vielversprechenden Weg zu einer qualitativ hochwertigen, effizienten und gut organisierten Patientenversorgung, die den WZWKriterien [3] genügt. Im Übrigen verwies der fmCh-Generalsekretär auf wichtige Organisationen, die sich in den chirurgischen und invasiven Disziplinen teilweise seit vielen Jahren in der Qualitätssicherung engagieren, so etwa die "Stiftung für Patientensicherheit» im Bereich der Prozessqualität und der «Nationale Verein für Qualitätsentwicklung in Spitälern und Kliniken» (ANQ) im Bereich der Ergebnisqualität. Das «Filetstück» sei in der Chirurgie indes die Indikationsqualität, so Trutmann, und auf diesem Gebiet seien vermehrt Anstrengungen nötig. Denn es nütze nichts, effizient und mit gutem Ergebnis zu operieren, wenn die Operation gar nicht nötig sei. Es gelte vor allem, falsche Anreize zu korrigieren. Ein Zertifikat für Indikationsqualität sei in Vorbereitung.

\section{Lebhafte Diskussionen}

In den Plenardiskussionen zu den Referaten zeigte sich, dass sowohl "Choosing-wisely-Listen" als auch Guidelines überwiegend als wichtig und nützlich eingestuft werden. Kritische Anmerkungen waren meist nicht grundsätzlicher Natur, sondern betrafen als problematisch oder verbesserungswürdig eingestufte Teilaspekte. So wurde beispielsweise in Bezug auf CWL bemängelt, die Empfehlungen seien insofern fragwürdig, als die beanstandeten Interventionen in der Schweiz ohnehin kaum von jemandem praktiziert würden. Angeführt wurde auch, bei den CWL handle es sich im Prinzip lediglich um eine willkürliche Zusammenfassung von Interventionen der Klasse III, die in der evidenzbasierten Medizin für «nicht indiziert, nicht nützlich, möglicherweise schädlich» steht. Diese Listen seien also nicht nötig, da die betreffenden Informationen schon vorhanden und zugänglich seien.

\section{SAQM}

Die Qualität sicherzustellen, ist eine Kernaufgabe von Ärztinnen und Ärzten. Die Schweizerische Akademie für Qualität in der Medizin SAQM wurde 2012 von der FMH gegründet, um Qualitätsaktivitäten zu vernetzen und zu koordinieren, Doppelspurigkeiten zu vermeiden und Synergien zu nutzen. Mehr zu den Aufgaben, Themenfeldern und Dienstleistungen der SAOM unter www.fmh.ch/saqm.html

seien für die praktische Medizin sehr wichtig, da es schwierig sei, den Verzicht auf eine Massnahme oder Intervention ohne breit abgestützte Positivliste zu kommunizieren. Auch Corinna Schaefer plädierte dafür, CWL und Guidelines ergänzend zu sehen.

Vertreterinnen der Pflegeberufe wiesen darauf hin, dass Guidelines bisher wenig interprofessionell ausgerichtet seien. Ärztliches Handeln sei aber selten isoliert, so dass eine vermehrte Einbindung anderer Professionen sinnvoll wäre. Auch in diesem Punkt war der Blick nach Deutschland aufschlussreich. Gemäss Corinna Schaefer werden dort verschiedene Berufsgruppen des Gesundheitswesens und über Patientenorganisationen auch die Patienten selbst zunehmend an der Leitlinienentwicklung beteiligt.

\section{Eine Aufgabe für die SAQM?}

Pietro Vernazza, Chefarzt Infektiologie und Spitalhygiene in St. Gallen, fasste in seinem Statement den breiten Konsens gut zusammen. Der Nutzen von Guidelines scheine allen klar zu sein, stellte er fest, aber die Schweiz sei einfach zu klein, um die von Corinna Schaefer beschriebenen Prozesse im Massstab 1:1 zu kopieren. Dies sei allein schon aus Ressourcengründen nicht möglich. Eine nachvollziehbare Aussage vor dem Hintergrund, dass gemäss Schaefer in Deutschland beispielsweise die Erarbeitung der Nationalen Versorgungsleitlinie «Diabetische Neuropathie» knapp 200000 Euro gekostet hat. Für Vernazza wie auch etliche weitere Votanten könnte die SAQM im Bereich der medizinischen Guidelines wichtige Aufgaben übernehmen, sowohl koordinativer Art bei ihrer Erarbeitung und Implementierung als auch bei der Evaluation ihres Nutzens. Christoph Bosshard nahm diesen Ball in seinem Schlusswort auf, zeigte sich grundsätzlich einverstanden, appellierte aber auch an den Willen zur Zusammenarbeit zwischen den verschiedenen Interessengruppen: «Wir müssen es gemeinsam anpacken.»

1 www.samw.ch/de/Projekte/Nachhaltige-Medizin.html

2 Selby K, Gaspoz JM, Rodondi N, Neuner-Jehle S, Perrier A, Zeller A, Cornuz J. Creating a list of low-value health care activities in swiss primary care. JAMA Intern Med. 2015 Apr 1;175(4):640-2.

3 Wirksamkeit, Zweckmässigkeit, Wirtschaftlichkeit gemäss Artikel 32 des Bundesgesetz über die Krankenversicherung. www.admin. ch/opc/de/classified-compilation/19940073/index.html 